\title{
Controverses et Maîtrise de l'information : Analyse et propositions méthodologiques
}

\section{Controversies and mastering information: analysis and methodological}

\author{
proposals
}

\author{
Orelie Desfriches Doria ${ }^{1}$, Catherine Dessinges ${ }^{2}$, Madjid Ihadjadène ${ }^{3}$ \\ ${ }^{1}$ Laboratoire ELICO, Université de Lyon, France, orelie.desfriches-doria@univ-lyon3.fr \\ ${ }^{2}$ Laboratoire MARGE, Université de Lyon, France, catherine.dessinges@univ-lyon3.fr \\ ${ }^{3}$ Laboratoire Paragraphe, Université Paris 8 - Saint Denis, madjid.ihadjadene@univ-paris8.fr
}

\begin{abstract}
RÉSUMÉ. Cet article présente une réflexion sur le rôle de la maîtrise de l'information dans l'analyse et la médiation de controverse, considérée comme un dispositif social innovant. II s'appuie sur une méthodologie originale d'analyse des controverses liée au contexte informationnel complexe actuel. Cet article développe notre méthodologie originale, synthétise les principaux résultats préliminaires de sa mise à l'épreuve auprès d'un public étudiant depuis 2014 et propose des pistes de réflexion pour l'affinement progressif de cette méthodologie d'analyse.

ABSTRACT. This article focuses on the role of information literacy in the analysis and mediation of controversy, considered as an innovative social measure. It is based on an original methodology for analyzing controversies related to the current complex information context. This article develops our original methodology, synthesizes the main preliminary results of its experiment with a student audience since 2014 and proposes perspectives for further development of this methodology.

MOTS-CLÉS. Controverse, Maitrise de l'information, Innovation sociale, Méthode d'analyse qualitative, Modélisation, Cartographie d'information, Écosystème informationnel, Information scientifique.

KEYWORDS. Controversy, Information Literacy, Social innovation, Qualitative analysis methodology, Modeling, Information cartography, Informational ecosystem, Scientific information.
\end{abstract}

\section{Introduction}

La mise en controverse d'une décision publique peut être vue comme un progrès social, car elle constitue une occasion de démocratiser les enjeux et les processus de décisions engageantes pour l'intérêt général d'une communauté. Ainsi, dans une controverse, des types d'acteurs variés peuvent intervenir, et il existe selon [LEM 07], une symétrie de principe entre les parties dans l'expression des arguments. Pour autant, nous considérons que cette symétrie de principe dans les interventions des acteurs s'opère dans un contexte informationnel complexe. Cet article présente une réflexion sur le rôle de la maîtrise de l'information dans l'analyse et la médiation de controverse, considérée comme un dispositif social innovant. Après avoir examiné le cadrage théorique, nous proposons une méthodologie originale d'analyse de controverse, visant à faire acquérir des outils conceptuels d'évaluation de l'information et d'esprit critique, notamment -mais pas seulement- à des publics d'étudiants. Ainsi nous considérons que les conditions de l'environnement socio-technique actuel nécessitent l'acquisition de compétences informationnelles, médiatiques, argumentatives et d'esprit critique, par les étudiants, (qui sont de jeunes citoyens, et les futurs parents des générations à venir) qui peuvent être enseignées à travers l'appropriation de cette méthodologie d'analyse et de représentation des controverses, qui sera décrite ci-dessous. Nous nous appuyons sur une expérimentation pédagogique menée sur plusieurs terrains dans l'enseignement supérieur, qui vise à tester, à amender, et affiner cette proposition de méthodologie d'analyse de controverses, au travers de la connaissance des arguments ainsi que des discours ou contraintes médiatiques. En termes de public, ces étudiants sont donc considérés comme des «repères» permettant d'identifier ce qui est difficilement appréhendé dans la méthodologie proposée, pour la rendre génériquement diffusable et partageable auprès de communautés plus largement investies dans des controverses aux enjeux variables. 
En résumé, après avoir rappelé le contexte informationnel actuel, et les approches les plus largement diffusées d'analyse de controverses, issues de la Théorie de l'Acteur-Réseau [AKR 06], nous présentons donc une méthodologie originale d'analyse de controverses. Puis nous préciserons rapidement les modalités de l'expérimentation menée depuis 2014, sur l'appropriation de ces approches innovantes de l'analyse de controverses, et quelques résultats et pistes de réflexion suite aux enquêtes réalisées sur ces terrains.

\section{Contexte informationnel et conceptions de la controverse}

\subsection{Evolution de l'environnement informationnel}

Depuis les récentes réformes des programmes scolaires, l'Education aux Médias et à l'Information (EMI) ainsi que l'Enseignement Moral et Civique (EMC) (citoyenneté, laïcité, morale, éducation aux valeurs de la république) ont été mis en avant par le Ministère de l'Education Nationale. Il s'agit d'une évolution nécessitée, notamment, par la place prépondérante prise par le numérique et les réseaux sociaux dans les pratiques d'information et culturelles des jeunes, mais également dans les interactions sociales au sein des réseaux de sociabilité. Si l'on s'en réfère à la condition humaine de l'Homo mediaticus, qui selon [FER 12], consiste à s'abstraire de la réalité, à s'absenter, à se replier ou à se réfugier dans ce qui s'apparenterait, selon nous, à des microcellules isolantes d'une ruche, il y aurait donc urgence à redonner du sens aux communs, au collectif, aux valeurs comme l'intérêt général ou le bien commun.

A l'ère numérique, n'importe quel citoyen peut en effet s'emparer d'un espace de parole sur le web, relayer une information, la commenter, la partager, sans avoir nécessairement acquis une certaine compétence médiatique consistant par exemple à avoir préalablement évalué la fiabilité de l'information partagée. Cette question de la fiabilité de l'information est d'autant plus centrale qu'Internet et les médias sociaux permettent une diffusion exponentielle et instantanée de toutes sortes d'informations, mais également de rumeurs, de fausses nouvelles etc. et que les pratiques informationnelles des jeunes sont aujourd'hui principalement tournées vers les réseaux sociaux numériques. Selon une étude conduite par trois chercheurs liés à l'Observatoire du Webjournalisme ${ }^{1}$, même si les jeunes ne sont pas dupes du potentiel manque de fiabilité des informations circulant sur les réseaux socio numériques, la grande majorité d'entre les 18-24 ans (73\%) préfère s'informer d'abord sur ces dispositifs. Sur ces plateformes, l'information n'y est de leur point de vue pas plus vraie que sur les médias traditionnels, mais elle a le mérite d'être divertissante et de se partager, des qualités qui montrent l'importance significative de la relation sociale pour les jeunes lorsqu'ils souhaitent s'informer.

La mise en place de ce nouvel écosystème de la consommation de l'information, lequel est fortement lié au développement d'une consommation croissante des écrans et multi-supports est allée de pair avec la montée des intox et des manipulations sur le web (création de fausses informations, fausses images, faux sites, fausses études) et à l'entrée des médias dans l'ère de la post-vérité. Ce concept fait référence à des circonstances dans lesquelles les faits objectifs auraient moins d'influence sur la formation de l'opinion que des mensonges assénés avec rhétorique et assurance. En témoignent la récente élection de Donal Trump aux États-Unis ou la campagne du «Brexit» en Angleterre. Dans le premier cas, " la post-vérité par indifférence », a été une arme massivement utilisée par Donald Trump pour se faire élire lors des présidentielles américaines : elle a consisté à ne pas toujours tenir compte de la vérité des faits dans les discours électoraux pour s'en tenir à des perceptions subjectives (au sujet du taux de fréquentation des meetings par exemple); l'autre forme de post-vérité qui peut s'ajouter à la précédente est la construction de fausses informations : l'un des arguments phares des partisans anglais du Brexit était de promettre qu'en cas de victoire le montant de l'adhésion hebdomadaire du Royaume Uni à l'Union européenne -soit 350 millions de livres sterling -serait utilisé

${ }^{1}$ http://www.slate.fr/story/142307/jeunes-consomment-information-facebook 
pour financer le système de santé publique du Royaume-Uni. Bien que démenti, ce chiffre a été associé à une vraie campagne de publicité et a eu un fort impact sur les électeurs [MON 17]. Ces exemples, qui ont par ailleurs été largement commentés et dénoncés par les institutions médiatiques et journalistiques s'inscrivent dans un contexte où les citoyens français se montrent très critiques à l'égard des médias et leur accordent une confiance limitée.

Cette crise de confiance des français vis-à-vis des médias et des journalistes s'est manifestée très largement à la lecture des enquêtes TNS-Sofres (février 2016) stipulant que plus de $70 \%$ des français ne font pas confiance aux médias. En cause, la croyance en la recrudescence des fausses informations et les théories complotistes ${ }^{2}$ mais aussi, selon Eric Mettout, le directeur adjoint de rédaction de l'Express délégué au numérique, « la méconnaisance du web» de la part de certains journalistes « qui ont pu parfois reprendre de fausses informations.» ${ }^{3}$. Pourtant à l'intérieur de la société démocratique, la déontologie de l'information précise que l'information doit être sourcée, vérifiée, libre et responsable ${ }^{4}$. Un enjeu démocratique et de citoyenneté pour lequel les supports médiatiques traditionnels, mais aussi les responsables politiques et les sites producteurs ou relayeurs d'informations doivent être vigilants à l'ère de la vitesse de propagation extrêmement élevée de l'information en ligne : un quart des événements se propagent en moins de 4 minutes. Ceci incite d'ailleurs au copié-collé de l'information ${ }^{5}$ et porte en soi le risque de supprimer à terme les incitations des médias à produire de l'information originale [CAG 17].

Face aux mutations induites par les technologies numériques dans la production d'information, mais aussi dans la manière de la consommer, et devant la recrudescence observée des dérives menaçant la démocratie de la part d'acteurs mal intentionnés ou peu scrupuleux, il semblerait qu'une prise de conscience collective soit désormais à l'œuvre. Aussi bien du côté des élites politiques qui ont fait de l'éducation aux médias numériques un projet d'enseignement prioritaire à l'école primaire et secondaire - on pense notamment à la loi du 8 juillet 2013 pour la refondation de l'École de la République qui inscrit officiellement l'éducation aux médias et à l'information (EMI) dans les programmes scolaires, du côté des associations familiales (UNAF), du côté de la société civile, des associations d'éducation populaire (Céméa, Ligue de l'enseignement, Jets d'encre etc.) que du côté des institutions elles-mêmes (France Télévisions, CNRS Images, collectif Éducnum, Réseau Canopé etc.) Le développement d'outils, plus ou moins collaboratifs, de fact-checking (firstdarftnews ${ }^{6}$; $\operatorname{crosscheck}^{7}, \operatorname{decodex}^{8}$ ) afin de lutter contre les imprécisions, les mensonges et les adeptes de la postvérité, révèle que l'évaluation de l'information, sa vérification sont aussi nécessaires en raison de cette accessibilité au statut «d'auteur», ou autrement dit de producteur d'information, donnée à tout un chacun, connecté. Ces dispositifs numériques appuient les mouvements qui développent de nouvelles modalités d'inscription des citoyens et de leur participation distribuée, dans l'innovation sociale, dans les décisions liées à l'économie ou au territoire, ou encore dans la co-construction des savoirs selon des démarches de sciences participatives. Concernant les sciences participatives, de nombreuses initiatives ${ }^{9}$ émergent, des réflexions se structurent autour des sciences citoyennes ${ }^{10}$, des groupes de travail ${ }^{11}$ contribuent à ces modalités innovantes de la construction des connaissances, et de nombreux projets ${ }^{12},{ }^{13},{ }^{14}$ ont été menés.

\footnotetext{
${ }^{2}$ https://www.franceinter.fr/societe/les-francais-sont-plus-complotistes-qu-on-le-pensait

${ }^{3}$ Eric Mettout cité par Justine Cantrel, La post-vérité pose question, Les clefs de la Presse, numéro 47.

${ }^{4}$ Partrick Eveno, Scoops, rumeurs, hoax, informations validées... Comment les identifier et vivre avec Webinar, Eureka.cc, 28 mars 2017

${ }^{5}$ Selon l'étude conduite par Cagé, Hervé et Viaud, 64\% de l'information chaude est du copié-collé

${ }^{6} \mathrm{https}$ ://firstdraftnews.com/

7 https://crosscheck.firstdraftnews.com/france-fr/

${ }^{8} \mathrm{http}: / /$ www.lemonde.fr/verification/

9 http://www.sciences-participatives.com/

${ }^{10} \mathrm{http}: / /$ www.allianceathena.fr/c/sciences-participatives

${ }^{11} \mathrm{http://www.alliss.org/}$

${ }^{12}$ https://www.camptocamp.org/

(C) 2018 ISTE OpenScience - Published by ISTE Ltd. London, UK - openscience.fr 


\subsection{La controverse comme innovation sociale}

Le Conseil supérieur de l'économie sociale et solidaire (CSESS) définit l'innovation sociale comme « des réponses nouvelles à des besoins sociaux nouveaux ou mal satisfaits dans les conditions actuelles du marché et des politiques sociales, en impliquant la participation et la coopération des acteurs concernés, notamment des utilisateurs et usagers. Ces innovations concernent aussi bien le produit ou le service, que le mode d'organisation, de distribution, dans des domaines comme le vieillissement, la petite enfance, le logement, la santé, la lutte contre la pauvreté, l'exclusion, les discriminations, etc. Elles passent par un processus en plusieurs démarches : émergence, expérimentation, diffusion, évaluation $»^{15}$. En définitive selon [BRO 16], qui retrace l'évolution historique de l'innovation sociale, on peut remarquer que les notions les plus souvent associées à ce concept sont les suivantes : le collectif, la concertation, les nouvelles pratiques, l'inclusion de minorités, le pouvoir d'agir (empowerment), l'ensemble s'inscrivant dans une « culture de la participation ».

L'ingénierie de la participation, ou de la concertation [BLO 08], quelle que soit la justification hypothétique adoptée de ses pratiques, notamment concernant la démocratie participative - la complexification du monde social, la division de la société dans des conflits moraux, la remise en cause des formes traditionnelles d'expertises, la mobilisation croissante des citoyens sur des causes mettant en jeu leur intérêt, ou encore le repli individualiste et la perte de confiance dans les institutions - on ne peut que constater la convergence des définitions de l'innovation sociale et des procédés de la démocratie participative. Ainsi en référence à ses effets, [BLO 08], définit la démocratie participative comme «l'ambition politique de faire participer à la prise de décision l'ensemble de ceux qu'elle est susceptible d'affecter » et dont l'objectif est « celui de la formation d'un jugement public éclairé parce que informé et issu d'une délibération large et approfondie. ».

Notre proposition consiste à analyser les controverses comme un phénomène de désaccord, en visant à terme à pouvoir structurer ces controverses à l'aide de la méthodologie présentée en partie 2 de cet article. Il s'agit donc de considérer la méthodologie en question comme une sous-classe des dispositifs de débat publics.

\subsection{La controverse comme un phénomène en soi}

En matière d'analyse et de représentation de controverses, il existe plusieurs approches d'après C. Lemieux [LEM 07]: une approche classique, historique, de la controverse qui agit " comme un révélateur » de la réalité socio-historique, et une approche initiée par les «Sciences and Technology Studies » qui considère les controverses comme des « phénomènes sui généris » permettant l'étude des transformations du monde social qu'ils produisent. Cette dernière approche a inspiré le courant de la sociologie des épreuves (aussi qualifiée de sociologie pragmatique), qui comprend deux sous-branches principales : l'anthropologie des sciences et des techniques développée sous les termes de «Théorie de l'Acteur-Réseau» par B. Latour et M. Callon, et la sociologie des régimes d'action de L. Boltanski et L. Thévenot [LEM 07]. La Théorie de l'Acteur-Réseau est issue des études menées par Bruno Latour, Madeleine Akrich et Michel Callon sur le champ scientifique et de l'innovation. Elle étudie l'interaction et la médiation entre des acteurs humains et non-humains qui forment un système : le réseau (ou les agencements sociotechniques). Dans cette approche les objets et les humains sont ainsi mis sur le même plan et considérés comme des actants, des médiateurs. Cette théorie s'appuie sur les notions d'attachement et de traduction [CAL 06]. D'autres approches tendent à analyser les cadres contraignant l'argumentation dans le dispositif du débat lui-même [CHA 07],[ELS 05], c'est-à-dire la manière dont l'organisation et le déroulement du débat influe sur l'expression des arguments.

\footnotetext{
13 http://vigienature.mnhn.fr/

14 http://lesherbonautes.mnhn.fr/

15 https://cnnumerique.fr/inclusion/ 


\subsection{La controverse, un événement social}

Nous précisons qu'à l'inverse de l'approche précédente qui va positionner l'analyste de la controverse comme un agent observant le phénomène, nos objectifs différent et sont liés à la définition suivante de la controverse que nous proposons, qui est d'ordre pragmatique et inspirée des travaux de Francis Chateauraynaud. Si l'on considère la possibilité de mise en controverse d'une décision publique comme un progrès social, voire comme une innovation sociale, elle est ainsi une occasion de démocratiser les enjeux et les processus de décisions engageantes pour l'intérêt général d'une communauté, et elle correspond à une co-construction de cette décision.

La controverse, dans notre approche, est donc considérée comme un événement social auquel les individus et les institutions prennent part, impliquant des niveaux et des natures de " concernement » variables [CHA 07],[CHA 11]. Ainsi un individu peut prendre part à un débat au titre du fait qu'il est témoin, ou victime par exemple, ou encore au titre de ses convictions politiques (écologistes par exemple). Selon nous, les controverses devraient aussi mettre en jeu des définitions de l'intérêt général et de l'intérêt particulier, dans la caractérisation des acteurs qui participent. Elles portent sur des sujets qui impliquent des connaissances et parfois un niveau de technicité élevé, et qui peuvent avoir un impact sur des choix concernant les modèles de société possibles (les controverses sur le transhumanisme ou sur l'euthanasie proposent ainsi une considération de l'humain et de la mort qui constituent des points de bascule vers des sociétés différentes). Elles se caractérisent aussi par des échanges d'arguments. Les conditions de déroulement du débat influant sur ces échanges, et par conséquent sur la nature des rapports de pouvoir impliqués dans les décisions. Enfin, elles constituent des dispositifs mettant en jeu des registres d'enjeux (technique, politique, scientifique, économique, voir figure 3), qui varient selon les acteurs qui s'expriment, et dont tous les degrés d'appréhension sont potentiellement à l'œuvre, des plus simples, aux plus élaborés : par exemple dans la mobilisation d'un « registre juridique » d'arguments, un juriste possède l'expertise qui lui confère plus de poids que lorsqu'un simple citoyen mobilise le même registre d'argument, avec un niveau de technicité potentiellement moins maitrisé.

Cette définition induit une vision de la controverse implique que tout citoyen (jeune, étudiant, enseignant, ou simple citoyen concerné) doit pouvoir prendre part aux débats, en maîtrisant tout au moins les aspects techniques (plus ou moins vulgarisés), mais aussi médiatiques, des questions posées. La connaissance des aspects techniques d'un débat peut se transférer à l'aide d'une médiation humaine, mais elle se réalise également au travers de la consultation de sources d'information numériques, caractérisées comme indiqué ci-dessus (en partie 1.1. de cet article), par leur hétérogénéité en termes de qualités : véracité, précision de l'information, citation des sources, fraicheur, caractéristiques des instances d'énonciation, cadrage médiatique, modalisation des discours. Tous ces éléments concourent à la construction des points de vue, et à la formation des opinions au sein de l'audience des médias numériques, en réseau ou non.

L. Blondiaux [BLO 01] affirme que « l'opinion publique ne peut plus être pensée comme la simple juxtaposition d'énoncés d'opinions individuelles ». En effet, il nous apparaît crucial de chercher à comprendre sur quels éléments, ou selon quelles modalités, se fait l'agrément entre les acteurs qui peuvent souscrire aux mêmes argumentations, ou à des morceaux de ces argumentations. Ainsi la méthodologie que nous présenterons en partie 2.3 de cet article utilise la catégorisation comme un outil de négociation et cherche à déterminer les natures des agréments entre les acteurs, en se basant sur des catégorisations partagées.

Ainsi la vision de la « controverse comme dispositif social innovant », au sens où elle se mettrait au service des démarches associées à la démocratie participative, décrites en partie 1.2., ne serait valable, selon nous, que dans le cas où les acteurs disposeraient de compétences équivalentes d'analyse des sources d'information, et de possibilités équivalentes de mobilisation et de maitrise d'outils conceptuels, méthodologiques et d'analyse médiatique ; le capital social, culturel et l'éducation reçue 
par les individus opérant des clivages et des inégalités dès l'entrée en scène des acteurs dans une controverse.

En somme, nous considérons que la véritable innovation dans la mise en œuvre de controverses ouvertes dans une société, consisterait à faire en sorte que les enjeux de pouvoirs liés à la maîtrise de l'information soient les moins déterminants possibles; à défaire l'emprise de la maîtrise de l'information sur la formation des opinions, ou autrement dit, à contribuer à la dissémination d'une vision de l'information literacy inclusive que nous développons dans la partie suivante.

\subsection{Information literacy et évaluation de l'information}

S'il est communément admis que l'acquisition de connaissances, et de compétences, dans la formation des opinions et la prise de recul nécessitent un temps long et une disponibilité cognitive, comment concilier la profusion d'informations liées au Web, l'injonction à la connectivité et à la publication permanentes, et le développement de compétences informationnelles et d'esprit critique ?

Dans la littérature abondante sur l'Information literacy, nous relevons trois axes principaux de réflexion. Le premier s'intéresse à la définition de ce concept, et au domaine couvert par ce champ [ELM 06]; [LOI 09]; [KOL 11]; [Le 12]. Cette partie de la littérature soulève des questions sur la convergence des différentes littératies : littératie informationnelle, littératie informatique, littératie de l'esprit critique, littératie documentaire, littératie médiatique, littératie numérique, littératie visuelle, et translittératie [Le 12]. Le second axe se focalise sur le développement d'outils, de standards et de méthodes d'évaluation des compétences en littératie informationnelle, comme [BRU 04] ou [ALA 00]. Le troisième axe se concentre sur une vision critique des deux premières approches, en incluant des réflexions sur la pédagogie pour la littératie informationnelle. En nous inspirant de cette dernière approche, et à la manière de [TUO 05] qui propose une vision socio-technique du champ, nous considérons que les compétences en littératie informationnelle ne constituent pas un jeu d'attributs qui peuvent être évalués et mesurés indépendamment des tâches pratiques à réaliser dans leur acquisition, et du contexte de leur mise en œuvre [TUO 05]. De plus dans la littérature française sur l'information literacy, [Le 09] propose une vision de ce concept en relation avec la citoyenneté et la maitrise de savoirs techniques, à laquelle nous souscrivons. Selon lui, le savoir technique compose une culture, qui entendue au sens de [SIM 89], a à voir avec les rapports de pouvoir. La vision de [Le 09] est également inspirée par les travaux de [STI 08] : la dimension de citoyenneté de la littératie informationnelle est basée sur une prise de conscience de la dimension duale des objets techniques qui sont décrits comme des « pharmaka » : ils peuvent autant constituer des solutions que des obstacles. Enfin, nous sommes également influencés par [SIM 09] qui introduit la notion de « culture numérique » qui implique une connaissance des codes culturels à l'œuvre dans le monde numérique, qui formatent l'accès à l'information et son interprétation.

Dans ce contexte, concernant les compétences médiatiques, nous nous situons dans la lignée des travaux de [OTT 10], repris par [LAN 15] au sein de leurs travaux sur l'éducation aux médias dans le champ de la recherche en Sciences de la communication en France ou au Canada. Ces derniers proposent une définition de la litéracie médiatique « articulée autour de deux axes : - 1) une compréhension des processus de production des messages médiatiques, ce qui comprend une connaissance des institutions médiatiques, des langages, codes et conventions des différents médias et une maîtrise, par la pratique, des processus de production de messages médiatiques ; - 2) l'acquisition des outils de pensée critique, qui permettent l'analyse des contextes et des processus de production, de diffusion et de réception des messages médiatiques. »

A ce titre, il est important d'avoir en tête qu'à l'ère du web 2.0 l'instance communicante peut être issue de la société civile ou d'individus isolés et donc ne dépendre d'aucune règle ou contrainte éditoriale d'une institution médiatique à laquelle elle appartiendrait (bloggeurs, contributeurs dans les forums et les wikis etc.). Pour autant, ces différents contributeurs, lorsqu'ils produisent des discours 
sur le web, c'est-à-dire quand ils les rendent disponibles et partageables de façon plus ou moins durable, procèdent à une activité de cadrage de l'information : sélection, organisation, structuration, modalisation de l'expression etc. Dès lors, la notion de cadrage médiatique inclut aussi bien celle des instances médiatiques relevant des médias traditionnels et de leurs déclinaisons numériques, que celle des instances de production d'un discours issu de plateformes collaboratives, de création, de partage, de relai, de diffusion : blogs, wikis, forums, plateformes de journalismes citoyens etc. Nous considérons également que le contrat de lecture dans les médias sociaux fait l'objet d'un implicite, plus ou moins repérable, d'autant que la culture numérique, entendu au sens de [SIM 09] peut posséder ses propres codes culturels et habitudes de lecture.

Il nous apparaît donc nécessaire d'être en mesure de repérer dans tous les types de discours médiatiques, ce qui relève des éléments factuels et ce qui relève de la modalisation du discours par l'instance communicante. Dans les médias traditionnels, « le fait est (...) issu de l'activité élémentaire de cadrage de l'expérience réalisée par chaque individu, c'est la création artificielle d'une discontinuité extraite d'un continuum temporel et spatial », [MAR 10].

Cette manière de considérer le cadrage médiatique d'un fait par les journalistes comme un construit, convoquant des normes, des valeurs, des représentations fait référence au courant de recherche nordaméricain des «media frames ». Il s'agit à travers cette expression de désigner la construction de sens opérée par les médias dont [GIT 80] est l'un des premiers analystes [MAR 10]. [GAM 89] ont poursuivi dans ce sens, et développé le concept de « panoplie interprétative »: « Ces panoplies associent d'une part des éléments de cadrage tels que métaphores, exemples, slogans, descriptions et images, et convoquent dans l'organisation du discours leurs éléments afférents de raisonnement tels que des relations de causes et de conséquences ou l'invocation de valeurs morales. Elles cherchent à mobiliser des résonances culturelles et prennent souvent appui sur des paroles légitimantes telles que celles de la science ou de la politique. » [MAR 10].

Lors de l'étude d'une controverse le traitement de l'information, l'analyse des sources et des arguments font l'objet d'une attention très particulière. Nous pensons alors que l'analyse (et la participation à) des controverses est une activité qui nécessite de mettre en œuvre des compétences médiatiques élargies, inspirées de celles mises en œuvre dans la réception d'un média traditionnel, et qui comporte un volet axé sur l'esprit critique. La diversité des acteurs impliqués et concernés par un sujet de controverse amplifie l'effet d'écho médiatique de certaines thèses et argumentations proposées et diffusées par les médias traditionnels et non traditionnels, en référence à la description de l'environnement informationnel présenté en partie 1.1 et à l'évolution concomitante des pratiques informationnelles. Il apparait donc indispensable d'analyser à la fois les sources médiatiques traditionnelles et celles issues des médias sociaux dans le cadre de l'étude des controverses. Cette dernière nous permettant de réinterroger les compétences médiatiques à l'œuvre lors de la réceptioninterprétation des textes, compétences entendues au sens de repérage du contrat de lecture, du cadrage médiatique du traitement d'un événement, et d'évaluation de la véracité, de la fiabilité, de la complétude, de la fraicheur et de l'exactitude de l'information. L'élargissement des sources émettrices d'information dans l'étude d'une controverse sur la base de publications textuelles accessibles sur le Web, permettrait donc de renforcer et d'approfondir ces compétences.

Nous proposons donc ici une démarche complémentaire des approches traditionnelles de l'information literacy, qui vise à donner des moyens et des outils aux étudiants, et aux citoyens plus généralement, pour leur donner des repères et des outils conceptuels dans l'exercice de leur esprit critique. Notre propos ne consistera pas à discuter des différentes définitions de ce concept, qui font varier sa formulation mais bien à présenter un cas d'étude sur la mise en œuvre de compétences médiatiques au sens large, et d'esprit critique dans l'étude de controverses. 


\section{Méthodologies d'analyse qualitative des controverses}

Parmi les projets les plus innovants en matière d'enseignement de l'esprit critique et d'éducation à l'information, les enseignements sur l'analyse des controverses semblent des plus prometteurs. S'appuyant la plupart du temps sur les travaux théorique de Michel Callon et Bruno Latour, c'est-àdire la Théorie de l'Acteur-Réseau (Actor-Network Theory), beaucoup de grandes écoles en France, comme SciencesPo, ou d'écoles d'ingénieurs comme L'ESIEE Paris, ou Telecom ParisTech, et certaines universités comme le département de Sciences Humaines et Sociales de 1'Université Paris 8, ont développé ce genre d'enseignements sur l'analyse et la représentation des controverses. Il s'agit en général de confronter les étudiants à la diversité des points de vue, tout en accompagnant l'analyse des controverses de l'application des principes de la théorie de l'Acteur-Réseau, tout en l'équipant des outils méthodologiques (comme les techniques d'entretiens) ou informatiques (comme les outils de crawling, de traitement textuels de corpus et de visualisation de ces données).

La méthodologie que nous proposons vise à donner des moyens d'analyse et de médiation de la controverse, ainsi qu'à organiser la médiation des connaissances nécessaires à une participation informée au débat, en considérant que le médiateur participe lui-même de l'intérieur au débat, et peut lui aussi avoir une opinion sur le sujet. Au-delà des différences en termes d'objectifs pédagogiques, et qui sont aussi liées à nos différentes définitions de la controverse, l'approche inspirée de la Théorie de l'Acteur-Réseau s'inspire des méthodes de text-mining ou de scientométrie (ou sociométrie) qui visent à révéler des aspects ignorés ou non attendus des débats, en se basant sur l'étude du lexique utilisé dans les échanges. A l'origine, ces méthodes visent à observer et à mesurer l'activité scientifique à travers l'observation des liens entre les textes, autrement dit la mesure du nombre des citations entre les acteurs publiant à propos d'un sujet. La méthodologie inspirée des approches de l'acteur-réseau est donc dépendante d'un corpus préalablement sélectionné de sources, et de l'analyse par des outils logiciels de text-mining. Enfin la représentation de l'analyse est réalisée, la plupart du temps, à travers un graphe de réseau des citations entre les acteurs, avec un outil comme Gephi ${ }^{16}$, par exemple [VEN 10]; [VEN 12].

Notre approche est plutôt axée sur une analyse qualitative et sociologique. Elle vise à comprendre, analyser, représenter, (communiquer sur) le contenu des arguments, les acteurs, les thématiques, les questions qui composent une controverse. Elle est basée, elle aussi, sur un corpus de sources, qui ne peut pas, par nature, être exhaustif, puisque les sources considérées proviennent du Web. Ainsi la sélection des sources est déterminante pour la portée de l'analyse de controverse considérée, et elle fait elle-même l'objet de questionnements, notamment sur la tentative d'embrasser et récolter des sources représentatives de toutes les positions des acteurs qui participent au débat. A ce titre, le repérage d'un acteur parti-prenante dans une controverse, mais qui ne s'exprimerait pas sur le Web, fait l'objet d'une attention particulière et relève de l'exercice de l'esprit critique de l'analyste. Une fois les sources récoltées, l'hypothèse de travail principale dont nous cherchons à évaluer la validité, consiste à utiliser un procédé de modélisation des acteurs, et des objets des arguments, puis à appliquer une typologie de registres d'arguments, pour l'analyse des controverses. La représentation cartographique accompagne et outille ce travail d'analyse et ne constitue pas une fin en soi. En effet, nous cherchons également à découvrir selon quelles modalités, la méthode à base de modélisation proposée est appréhendable par différents types de publics, et à ce titre, nous considérons qu'elle peut s'appliquer en dehors de l'usage de tout outil informatique, en tant qu'outil conceptuel d'objectivation partielle des arguments. La maîtrise des outils de modélisation utilisés dans ce cadre vise à acquérir le mode de raisonnement correspondant. C'est ce que nous présentons dans la suite de cet article.

Aussi bien dans la méthodologie inspirée de l'Acteur-Réseau que dans celle que nous proposons, le problème de l'objectivité (ou autrement dit de la subjectivité) de l'analyste est présent, notamment dans le choix des termes inclus dans l'étude du corpus à l'aide d'outils d'analyse automatique de textes

\footnotetext{
${ }^{16}$ https://gephi.org/

(c) 2018 ISTE OpenScience - Published by ISTE Ltd. London, UK - openscience.fr
} 
(pour les tenants de l'Acteur-Réseau), dans le choix des outils logiciels, et leur paramétrage, et enfin, dans le choix des dispositifs de représentation et de leur fonctionnement algorithmique également.

\subsection{Présentation de l'expérimentation}

De octobre 2014 à janvier 2017, nous avons conduit une expérimentation pédagogique, dans le cadre d'un enseignement sur les controverses dispensé par le premier auteur de cet article, avec deux publics étudiants, l'un à l'ENSCI-Les ateliers, l'autre à l'Université Paris 8 - Saint-Denis. Pour consulter quelques résultats préliminaires, on pourra se référer à [DES 16]. A ce jour, nous avons réalisé trois occurrences de cet «atelier controverses», dont deux avec des groupes d'étudiants en Master 2, Gestion Stratégique de l'Information, à l'Université Paris 8, et l'un à l'ENSCI-Les Ateliers (Ecole de Design). Le cours consiste à développer progressivement les compétences liées à la méthodologie présentée en partie 2.4 , de recherche d'information, de collecte et de classement des sources, d'analyse des sources sélectionnées, de modélisation de la controverse, et enfin de représentation cartographique pour synthétiser l'ensemble de l'étude. En moyenne, l'atelier se déroule sur une durée qui correspond à une semaine entière de cours, alternant les apports théoriques et les mises en pratique à chaque étape.

\subsection{Méthodologies d'observation et de collecte de données}

La démarche adoptée ici, qui vise à évaluer dans quelle mesure la méthodologie présentée en partie 2.4 de cet article est appropriable par différents publics, consiste à récolter des données qualitatives en utilisant plusieurs méthodologies, de manière complémentaire: observation participante, enregistrements, retranscription, questionnaires, analyse des travaux des étudiants. Ainsi, nous collectons des données pendant l'atelier selon les principes de l'observation participante, en prenant des notes sur les questions et problèmes rencontrés par les étudiants dans l'appropriation de la méthode, ainsi qu'en faisant des enregistrements sonores retranscrits en intégralité, pendant les phases de tâtonnements et de réalisation de l'analyse, afin de conserver des traces fidèles des problèmes qu'ils rencontrent, notamment concernant la modélisation. Nous procédons également à un questionnaire après l'atelier dans les deux mois qui le suivent, afin d'évaluer les conditions de l'atelier, et les acquisitions de compétences. Enfin une fois les travaux rendus, nous étudions les supports cartographiques produits, qui sont rassemblés dans des petits sites Web. Il est important de noter que la méthodologie est présentée aux étudiants comme un work-in-progress, qui continue donc d'évoluer en prenant notamment en compte leurs retours, et apports potentiels. Nous les invitons également à sortir d'une vision simpliste qui n'intègrerait que des catégories d'acteurs de type Pour, Contre, Neutre, qui correspond aux catégories du sentiment analysis disponibles dans les outils numériques d'évaluation des ressentis des internautes sur des sujets d'actualité comme notamment dans 1'outil Social Mention ${ }^{17}$.

\subsection{Méthodologie de modélisation et de cartographie pour l'analyse de controverse}

En premier lieu, il convient de préciser que dans cette expérience pédagogique, l'objectif pour les étudiants est de jouer le rôle de médiateur de la connaissance nécessaire pour prendre part à une controverse dans l'espace public. Ce rôle implique des questions de positionnement et de subjectivité. En effet, comment peuvent-ils être assurés de ne pas introduire de biais dans l'analyse, quand le sujet de la controverse peut les concerner, à juste titre puisqu'il s'agit de questions de société, en référence à la définition de la controverse présentée en partie 1.4. Par quels moyens peuvent-ils éviter les potentielles distorsions des points de vue à l'œuvre dans les arguments confrontés dans la controverse ? Ainsi, durant le processus d'analyse, les étudiants doivent prendre conscience de leurs propres opinions, afin de tenter de présenter les éléments de la controverse dans une neutralité relative, c'est-àdire le moins possible influencée par leurs propres convictions.

\footnotetext{
${ }^{17}$ http://www.socialmention.com/ 
Les modèles se caractérisent par le fait qu'un « modèle est toujours plus simple que l'objet, le phénomène ou le processus qu'il est supposé représenter et expliquer. C'est un moyen servant tant à la représentation qu'à l'étude d'un phénomène. » [WIL 96]. Ce que la pratique de la modélisation vise à réaliser est une réduction de la complexité en se focalisant sur certains aspects, en fonction de certains buts. Il s'agit donc de conceptualiser les entités du domaine et les interactions en travaillant sur une abstraction des éléments du débat. Ainsi, tout au long de ce que nous appelons la modélisation du débat, il y a trois classes d'objets auxquels nous prêtons une attention particulière : les acteurs, les objets du débat, et les arguments. Dans l'élaboration du modèle des connaissances liées à une controverse, il est nécessaire de s'intéresser au concept plutôt qu'à son occurrence dans le débat, au moins à l'étape de définition de ses propriétés. C'est précisément à travers ce processus de décontextualisation des objets du débat, pour leur modélisation, que l'étudiant, ou le citoyen, met en œuvre la prise de recul nécessaire à la mise à distance de ses propres opinions sur le débat.

Dans un premier temps, la modélisation permet de caractériser les acteurs en fonction de la place qu'ils occupent dans le champ social, de leurs groupes sociaux d'appartenance et de leurs cultures épistémiques en jeu dans la définition d'un univers de référence, qui contribue aux panoplies interprétatives de leurs énoncés dans le débat, (ce concept a été présenté en partie 1.5 de cet article).

\subsubsection{Propriétés des acteurs de la controverse}

En fonction des caractéristiques propres à cette catégorie des acteurs (personnes physiques), nous avons élaboré un jeu de propriétés qui nous paraissent pertinentes dans le cadre de l'analyse des controverses en général. Elles proviennent de l'analyse de l'expérience de débat réalisée dans le cadre du projet RisCom Gaz de Schiste (ISCC/CNRS-Cnam), piloté par M. Letté, et elles sont présentées ciaprès. Nous souhaitons préciser ici qu'il est difficile en général d'obtenir l'ensemble des informations qui permettraient de renseigner chaque propriété. Il s'agit donc ici d'élaborer un modèle idéal des informations qui permettraient de décrire les acteurs qui interviennent dans un débat, et qui est mis en œuvre en fonction des conditions réelles du contexte :

- le statut : renvoie au statut de l'acteur dans le débat, et a à voir avec sa légitimité et son pouvoir, par exemple : victime, expert, journaliste, scientifique, militant ;

- la catégorie : il s'agit de la sphère sociale d'appartenance sociale convoquée lors de l'intervention dans le débat, par exemple, Scientifique et technique, Economique et marchand, Juridique et administratif, Social, Socio-politique et citoyen ;

- les objectifs génériques des interventions de l'acteur dans le débat : par exemple, production de connaissances, exploitation des connaissances, intérêt général, régulation juridique, la mise en débat et la participation citoyenne ;

- le rattachement à une organisation : il s'agit de l'appartenance à des groupes sociaux identifiées, comme par exemple le « collectif non aux forages »;

- les valeurs et pratiques : par exemple, la construction collective de l'expertise, ou la réappropriation du dialogue social, ou encore le profit de l'entreprise, ou bien la pérennité des institutions ;

- la culture épistémique : il s'agit de la «culture métier» de référence d'un intervenant d'un débat, par exemple, le militantisme, le syndicalisme, la direction d'entreprise, les responsabilités administratives, la culture scientifique ;

- le « concernement» : cette notion provient des travaux de Chateauraynaud (2011) et interroge la motivation au titre de laquelle l'acteur est engagé dans le débat : par exemple, par solidarité, parce qu'il est témoin, au titre de son engagement dans la vie de la commune, parce qu'il est écologiste ... 
- le registre émotionnel : cette propriété peut consister à reprendre ou extraire des expressions stéréotypées issues de verbatims, par exemple : « traumatisme », " électrochoc », " sortir de son confort ";

- les objectifs personnels de la participation de l'acteur au débat : collecter de l'information, les archiver, informer, fédérer, réguler, etc.

- la définition de l'intérêt général et particulier par l'acteur : en référence à notre définition de la controverse (voir partie 1.4.), nous pensons que ces aspects mériteraient une étude à part entière, afin de rechercher les convergences et les divergences sur ces définitions qui motivent en grande partie l'intervention des acteurs, en termes d'engagement dans les débats, selon nous.

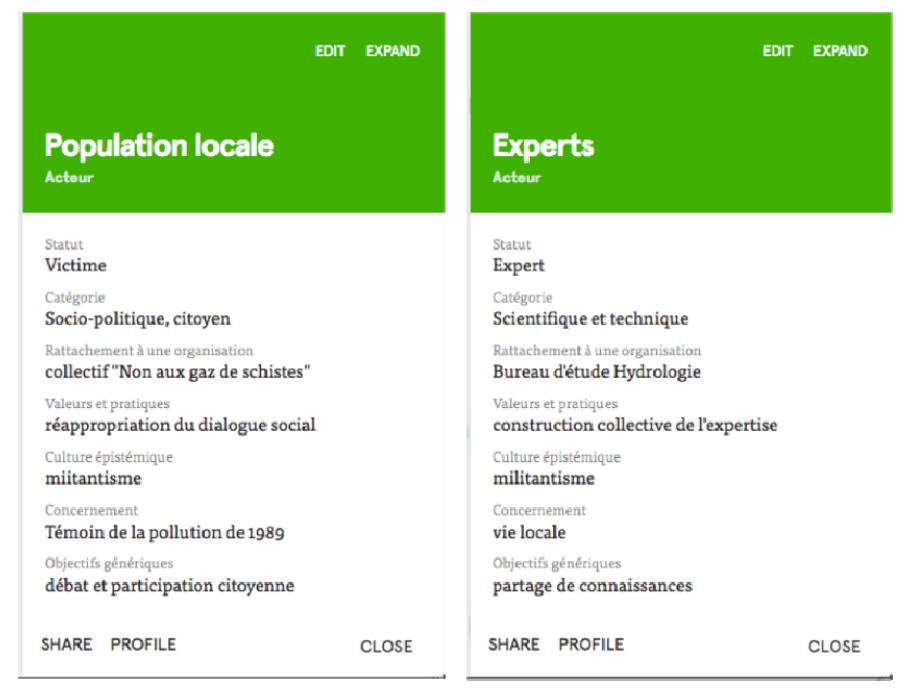

Figure 1. Représentation des propriétés de deux types d'acteurs dans GraphCommons

La figure 1 présente la matérialisation des propriétés présentées ci-dessus dans une interface logicielle associée à une cartographie, dans l'outil GraphCommons ${ }^{18}$.

\subsubsection{Objets du débat, et leurs propriétés}

Dans un second temps, la méthodologie consiste à faire émerger les objets du débat en tentant de définir leurs propriétés objectives en dehors de tout discours d'information tout en s'appuyant sur ses propres connaissances empiriques du monde, ses propres représentations et en s'inspirant de la technique de modélisation-objet. Ces objets du débat correspondent à l'objet des arguments dans l'approche de [CHA 11], qui préconise d'entrer dans l'analyse d'un débat par les arguments, en les considérant comme composant "les unités de signification élémentaire qui rendent possibles les comparaisons et analyses à partir de matériaux foncièrement hétérogènes ", et qui propose de penser l'argumentation en termes de portée :

- Qui porte un argument ? (acteur, énonciateur)

- Sur quoi porte-t-il ? (son objet, sa thématique)

-Quelle est sa portée ? (son extension, ses conséquences).

Ainsi, dans une controverse sur le gaz de schistes, divers objets interviennent comme des « forages », des « pollutions », des « risques »... chacun de ces objets est un objet du débat. Ils sont repérés dans les énoncés à l'aide de la technique qui consiste à repérer les éléments de la modalisation du discours : adjectifs superlatifs (inadmissible, formidable), adverbes, (peut-être, sans doute, probablement) conjugaison (conditionnel), verbes (devoir, pouvoir, sembler, paraitre, prétendre) etc. Par opposition

${ }^{18}$ https://graphcommons.com/

(C) 2018 ISTE OpenScience - Published by ISTE Ltd. London, UK - openscience.fr 
aux éléments de modalisation du discours, les éléments qui peuvent constituer des objets du débat seront considérés comme les éléments factuels dans les énoncés, donc schématiquement, ce sont les éléments non modalisés. Dans le même sens, lors du projet RisCom - Gaz de Schistes, évoqué précédemment, nous avons pu réaliser la carte suivante, présentée en figure 2, qui décrit les définitions par les parties prenantes au débat, de la notion de risques dans l'exploration et l'exploitation des gaz de schistes. Cette carte permet d'observer une grande diversité des vues sur cette définition, et de rendre observable le peu de recoupement entre les manières d'appréhender ces risques.

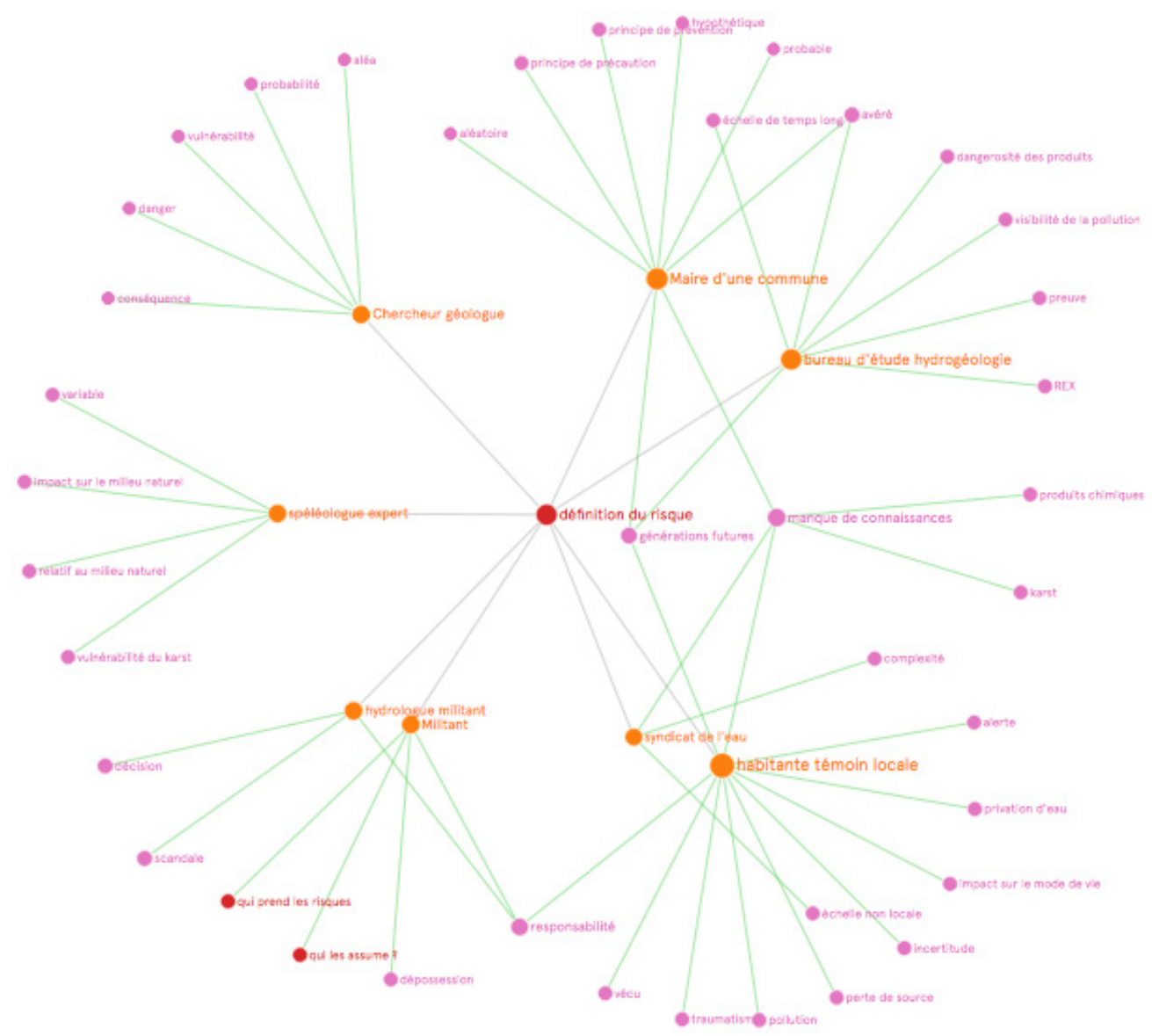

Figure 2. Représentation de la diversité des définitions de la notion de risques dans GraphCommons

Une fois le repérage des objets réalisé, la méthode consiste à s'intéresser à ces éléments du point de vue conceptuel, c'est à dire à chercher ce qui les caractérise en général en dehors du contexte de la controverse étudiée. Ainsi les concepts suivants pourraient avoir pour propriétés génériques :

- forage : une profondeur mesurable, une localisation, un coût, une technique utilisée, des produits chimiques l'accompagnant, un industriel qui le réalise, une ressource recherchée ;

- pollution : une ou plusieurs mesures, une zone géographique concernée, une ou des substances impliquées, des effets déjà démontrés, une durée, des mesures de retraitement ;

- risque : des ressources naturelles affectées, un responsable, une quantification, des victimes, une zone géographique plus ou moins étendue.

En termes de structuration et d'animation d'un débat, cette étape correspondrait à la mise en commun de définitions divergentes des objets du débat, la définition de leurs propriétés respectives étant considérées comme une partie du débat en lui-même. Ainsi, plusieurs groupes d'acteurs se confrontant dans un débat pourraient définir les objets et leurs propriétés selon des points de vue différents, et produire ainsi des cartes reflétant leur vision des problèmes. Nous pensons que cette pratique présenterait l'avantage de clarifier les termes du débat, et de les documenter et d'en permettre une lecture approfondie. Au cours d'un débat il serait donc possible de déterminer les propriétés génériques d'un objet « forage », et ensuite de déterminer les informations manquantes, en fonction des 
acteurs présents, ainsi que les divergences de définition de ces objets à la manière de ce qui est présenté dans le tableau 1 sur un objet « forage » selon deux types d'acteurs :

Selon un acteur industriel

Un coût : $100000 €$

Une localisation : coordonnées GPS

Un effet sur l'environnement : NUL

Procédé technique : fragmentation hydraulique

Substance additionnelles : soumis au secret industriel
Selon un acteur « habitant local»

Un coût : inconnu

Une localisation : coordonnées GPS

Un effet sur l'environnement : risque de pollution de l'eau, destruction du milieu naturel

Procédé technique : fragmentation hydraulique

Substance additionnelles : soumis au secret industriel

Tableau 1. Description des propriétés d'un forage pour deux acteurs d'un débat sur le gaz de schistes

\subsubsection{Analyse des arguments}

L'étape suivante consiste, une fois les acteurs et les objets des arguments décrits sur la base de discussion autour de leurs propriétés, à associer aux arguments un registre principal qui caractérise l'univers (ou la panoplie interprétative) auquel il fait référence. La figure 3 présente une liste non exhaustive de ces registres, indépendants les uns des autres. Chaque case de ce tableau correspond à un registre qui peut servir à caractériser un argument. Cette association des arguments à ces registres permet de donner une lecture synthétique de la controverse en observant les rattachements principaux à ces registres, des arguments échangés au cours d'une controverse.

\begin{tabular}{|l|l|l|l|}
\hline Economique & Idéologique & Sociologique & Territorial \\
\hline Juridique & Environnemental & Social & Psychologique/Emotionnel \\
\hline Politique & Sanitaire & Symbolique & Scientifique \\
\hline Géostratégique & Identitaire & Éthique & Géomorphologique/Géologique
\end{tabular}

Figure 3. Liste des registres des arguments, non exhaustive

La cartographie présentée en figure 4, présente le problème suivant dans les questions liées aux gaz de schistes: les firmes industrielles et les experts se contredisent quant à l'utilisation de produits chimiques lors des forages réalisées avec de la fragmentation. Il s'agit d'un registre technique qui est présenté en violet sur la carte. Le registre économique indiqué en vert, correspond au fait que les populations locales ne peuvent pas payer des analyses de ces éventuels produits parce qu'elles coûtent trop cher. Le registre administratif (en jaune) est convoqué à propos de la demande des populations locales faites auprès de l'administration, pour obtenir des informations au sujet de ces produits. Les firmes imposent le secret industriel à l'administration et elle ne peut, de ce fait, donner des renseignements aux populations locales, c'est alors le registre juridique (en bleu) qui est employé. Enfin, les populations locales qui jugent que ce refus pose un problème de démocratie, est matérialisé comme un registre politique de couleur rouge. Ainsi dans cette carte, la fragmentation, les produits chimiques, les analyses, les pollutions, le secret industriel correspondent à des objets d'arguments. 


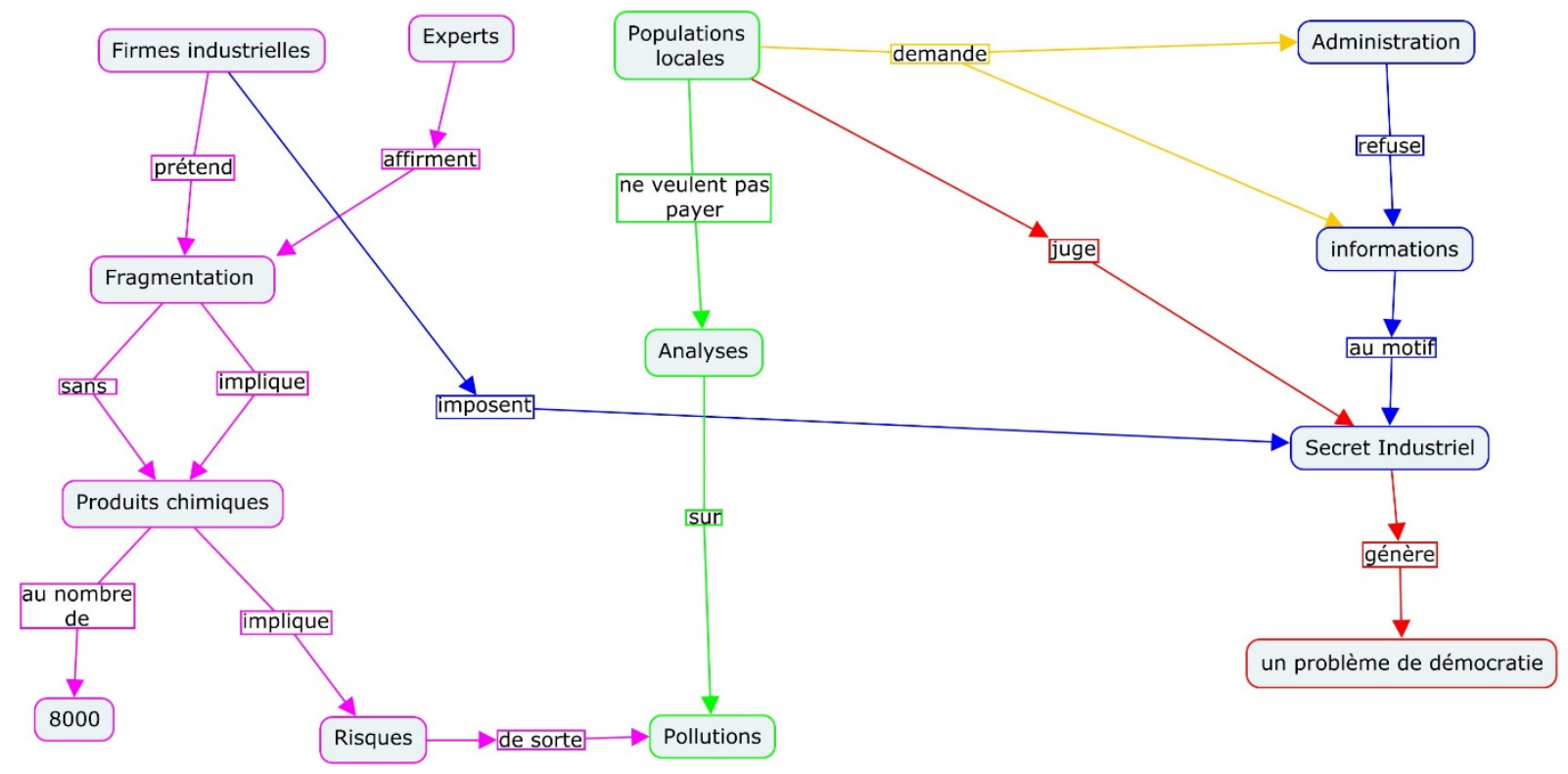

Figure 4. Cartographie des arguments par registres sur la question des « Forages et du secret industriel »

\section{Résultats et discussions}

Au-delà des nombreuses analyses qu'il convient de mener sur les matériaux de données récoltées, mais qui excèdent le cadre de cet article, nous souhaitons ici mettre l'accent sur deux questions qui nous intéressent particulièrement, à propos de l'évaluation de l'information scientifique et de la remise en cause à l'égard des institutions d'une part, et à propos de la nature des citations et du rapport à la preuve d'autre part. Les éléments présentés ci-après constituent des pistes de réflexion, que nous développerons davantage dans des publications complémentaires à cet article.

\subsection{Remise en cause des institutions et évaluation du travail scientifique}

Le constat de la remise en cause des institutions est assez largement répandu et il n'est pas si récent [BLO 11]; [BLO 08]; [SIN 08] ; [SIN 11]; [OCT 09]; [MER 17] ; [COR 17]. Il est notamment mis en perspective dans un rapport ${ }^{19}$ du Cevipof en 2011, qui porte sur la confiance/défiance vis-à-vis, notamment, des institutions politiques. Ce dernier indique que l'évocation de la politique en 2011, provoque un sentiment de méfiance à hauteur de $39 \%$, d'ennui pour $12 \%$, et de dégoût pour $23 \%$ des enquêtés. [SIN 11] tente d'en déceler les causes, parmi lesquelles il recense les suivantes : la perte de pouvoir des Etats vis-à-vis de l'économie mondialisée ; la perte de puissance de l'idéal du progrès ; ou encore l'affaiblissement de la justification de la délégation des décisions aux politiques ou aux scientifiques, dans un monde un incertain.

Lors de l'atelier à l'Université Paris 8 en 2017, l'un des groupes d'étudiants a mobilisé une source scientifique, publiée dans une revue, sans être en mesure d'évaluer la qualité et les apports de cet article. En effet, certaines sources scientifiques peuvent contenir des informations obsolètes, ou qui n'auraient pas été suffisamment vérifiées ou recoupées par d'autres études. Les étudiants ont ici considéré que si quelque chose est publié, cela est vérifié et vrai, sans distinction. Cela a à voir avec l'écosystème des publications scientifiques, qui peut apparaître opaque aux yeux du grand public. Celui-ci n'est pas nécessairement au fait des pratiques de publications dans la recherche, et de la valeur variable associée aux éditeurs et lieux de publications. De plus, il est important de souligner que la plateformisation du web modifie les frontières-contours de la confiance puisque la notion de hiérarchie de sources d'information, y compris scientifique, s'estompe. Dans un contexte où l'augmentation exponentielle des publications rend la réception difficile, les usagers se trouvent confrontés à un manque de différentiation sur les réseaux sociaux, entre l'information de bonne et de mauvaise qualité

\footnotetext{
${ }^{19}$ http://www.cevipof.com/fr/le-barometre-de-la-confiance-politique-du-cevipof/rapports/notes/ 
[QIU 17]. Pour autant, le constat du déclin de la confiance dans les autorités scientifiques n'est pas clair : les enquêtes du Pew Research Center en $2017^{20}$ montrent que la confiance dans les scientifiques est très élevée mais que les pratiques de recherche de l'information scientrifique par les usagers se font principalement sur les réseaux sociaux [BRO 13] ; [GAU 10].

Les caractéristiques spécifiques des connaissances scientifiques, tout au moins dans les champs comme la biologie ou la physique, (d'être construites, en référence à des paradigmes, leur besoin de vérification, et de reproduction des études pour leur validation, par exemple) sont mal connues des étudiants et leur culture scientifique, actuellement, ne leur permet pas de savoir évaluer la fiabilité de l'information scientifique. Ainsi, l'étudiant type dans ce cours - et par extension le citoyen en général - ne possède pas les connaissances scientifiques pour évaluer par lui-même le contenu des éléments publiés. L'idée ici consisterait à resituer la nature de l'information scientifique comme un type d'information dont il faut chercher à évaluer la fiabilité au même titre que d'autres types d'informations. L'isolement ou la reconnaissance d'un scientifique par sa communauté scientifique, peut, par exemple constituer un indicateur de la qualité de ses publications. D'autres sortes d'indicateurs pourraient être envisagées, comme, par exemple, un degré de validation basé sur un degré de reproduction des études, ainsi que des descriptions des méthodologies employées, et des degrés de rigueur de celles-ci. Les éléments présentés ici sont des pistes de réflexion, et ne peuvent se substituer à un travail d'investigation plus complet sur ces questions. Pour autant, il nous semble donc qu'il y a un véritable besoin de formation sur les méthodes et les caractéristiques de la connaissance scientifique pour donner des indicateurs aux citoyens afin qu'ils aient des repères dans l'évaluation de l'information liée aux controverses sociotechniques.

\subsection{Citations et " evidentiality "}

Dans les analyses de controverses inspirées par les approches de l'acteur-réseau, les graphes sont produits automatiquement par des algorithmes à partir de la collecte et des traitements linguistiques appliqués sur les corpus, notamment en matière de citation entre les sources. Selon nous, les arguments développés par les acteurs, ni les connaissances techniques requises ne sont généralement présentés pour parvenir à prendre part à un débat en étant (in)formé. Les graphes de citations entre les acteurs sont accompagnés de textes explicatifs, mais la nature des liens sémantiques n'est pas explicitée dans ces derniers. Nous considérons que ce type d'approche réalise des photographies successives de l'état de la participation des acteurs au débat, afin d'étudier le "phénomène controverse » en lui-même.

Pourtant la nature de la citation, sa motivation, ou autrement dit, la question du rapport à la preuve pourraient faire l'objet d'une description plus détaillée. En effet, dans la littérature scientifique, la citation des travaux des pairs peut être motivée par plusieurs éléments distincts : un apport précédent à l'étude menée, ou encore, une volonté de contredire ou discuter des propositions, par exemple. Par ailleurs, la source elle-même peut faire l'objet de divers degrés de fiabilité, et les travaux de [RUB 10], qui propose un modèle de l'évaluation de l'incertitude de sources d'information médiatiques, sont particulièrement éclairants sur ce point. Enfin, dans la manière de référer à une source, la langue ellemême donne des indicateurs de la fiabilité accordée par un locuteur à cette source. Ainsi, il existe un courant dans le champ de la linguistique, des evidentiality studies, qui a débuté dans les années 80 aux Etats-Unis [DEN 94], et qui permettrait d'analyser la source d'une information ainsi que sa fiabilité, sur la base d'une typologie de marqueurs évidentiels : « un marqueur évidentiel signale de quel type d'indices on dispose quant à la fiabilité de l'énoncé dans lequel ce marqueur est utilisé » [DEN 94]. De nombreuses typologies liées à cette notion d'evidentiality existent, et il nous apparaîtrait inadapté ici d'en faire l'inventaire. Pour autant il nous semble que ces travaux présentent un potentiel certain pour la construction d'indicateur de la sorte de rapport à la preuve mis en jeu dans les énoncés. Une introduction complète à cette notion est réalisée par [ROO 01]. Pour préciser les termes de cette

\footnotetext{
${ }^{20}$ http://www.pewresearch.org/fact-tank/2017/04/06/public-confidence-in-scientists-has-remained-stable-for-decades/ (C) 2018 ISTE OpenScience - Published by ISTE Ltd. London, UK - openscience.fr 
approche, nous utilisons ici un exemple, qui consiste à étudier quel est le rapport à la source de l'information en tant que preuve d'un énoncé. Si un locuteur dit :
a) J'ai vu Mr Duchamp, il est à l'université.
b) J'ai vu la voiture de Mr Duchamp dans le parking, il est à l'université.
c) On m'a dit que Mr Duchamp est à l'université.
d) Mr Duchamp est probablement à l'université.
e) Il me semble que Mr Duchamp est à l'université.
f) Mr Duchamp est à l'université.

La phase a) s'appuie sur une perception qui témoigne d'un fait, tandis que la phrase b) s'appuie sur un indice utilisé pour réaliser une inférence. La phrase c) trouve sa source auprès d'une autre personne, tandis que la phrase d) correspond à une supposition, tout comme la phrase e). Enfin la phrase f) correspond à une affirmation, dont la source n'est pas mentionnée.

A la lumière des pistes proposées ici, il apparait qu'un travail de précision et de typologie reste à mener sur la qualification de la nature des citations, et la nature des liens entre des sources (hypertextes notamment), dans la direction des travaux menés par [SAE 12]; [SAE 15] sur la rhétorique des liens hypertextes, ainsi que sur la manière de référer à une source comme preuve d'un énoncé. Ces travaux pourraient outiller l'évaluation de l'information, en procurant aux étudiants et aux citoyens, des repères conceptuels, applicables en dehors même de l'utilisation des outils numériques.

\section{Conclusion}

Après avoir rappelé le contexte informationnel actuel, notamment le rôle des réseaux sociaux dans les pratiques d'information, nous avons présenté une méthodologie originale d'analyse des controverses, qui vise l'acquisition, pour les étudiants du cas d'étude présenté, d'outils conceptuels d'évaluation de l'information. Cette expérimentation que nous menons depuis 2014, avec différents groupes d'étudiants, a été présentée rapidement. Celle-ci nous permettra, à terme, de construire des modes d'appropriation et de vulgarisation de la méthodologie, pour le grand public, dans le but d'en faire un outil conceptuel mis à disposition des acteurs citoyens qui veulent prendre part aux controverses. Ces possibilités de généralisation nécessitent encore des analyses approfondies de nos données, ainsi que des expérimentations complémentaires. Les résultats et pistes de réflexion présentés préfigurent les affinements progressifs prochains de la méthodologie qualitative d'analyse des controverses.

\section{Bibliographie}

[AKR 06] AKrich M., CAllon M., LATOuR B. (Eds.), Sociologie de la traduction: Textes fondateurs. Presses des Mines, Paris, 2006.

[ALA 00] AMERICAN LIBRARY ASSOCIATION, Information literacy competency standards for higher education, 2000.

[BLO 17] BLONDIAUX L., Le nouvel esprit de la démocratie-actualité de la démocratie participative. Le Seuil, 2017.

[BLO 08] BLONDiAuX L., « Démocratie délibérative vs. démocratie agonistique? », Raisons politiques, n², p. 131-147, 2008.

[BLO 01] BLONDIAUX L., « Les théories contemporaines de l'opinion publique: un retour aux « classiques » ? », Hermès, La Revue, Vol. 3, n 31, p. 9-20, 2001.

[BRO 13] BROSSARD D., « New media landscapes and the science information consumer. » Proceedings of the National Academy of Sciences, vol. 110, no Supplement 3, p. 14096-14101, 2013.

[BRO 16] BROWNE P. L., « La montée de l'innovation sociale. » Quaderni, n², p. 55-66, 2016. 
[BRU 04] BRUCE C. «Information Literacy as a Catalyst for Educational Change. A Background Paper. » In: DANAHER, P. A. (eds) Proceedings "Lifelong Learning: Whose responsibility and what is your contribution?", the 3rd International Lifelong Learning Conference, p. 8-19, Yeppoon, Queensland, 2004.

[CAG 17] CAGE J., HeRve N., Viaud M.L., L'information à tout prix, (No. hal-01521888), 2017.

[CAL 06] CALlon M., FerRary M., "Les réseaux sociaux à l'aune de la théorie de l'acteur-réseau », Sociologies pratiques, $\mathrm{n}^{\circ} 2$, p. 37-44, 2006.

[CHA 11] Chateauraynaud F., Argumenter dans un champ de forces, Essai de balistique sociologique, Editions Petra, Paris, 2011.

[CHA 07] CHATEAURAYNAUD F., « La contrainte argumentative. Les formes de l'argumentation entre cadres délibératifs et puissances d'expression politiques ", Revue européenne des sciences sociales, TOME XLV, n¹36, p. 129-148, 2007.

[COR 17] CORDY J., La révolution des médias sociaux: incidences politiques et sécuritaires, Projet de rapport auprès de l'Assemblée parlementaire de l'OTAN, Commission sur la dimension civile de la sécurité, 2017.

[DEN 94] Dendale P., TASMOwski L., « Présentation: l'évidentialité ou le marquage des sources du savoir », Langue française, p. 3-7, 1994.

[DES 16] DESFRICHES DORIA O., IHADJADĖNE M., « Mastering Information and teaching controversies: an exploratory study », in Kurbanoğlu S. et al. (eds), Information Literacy: Key to an Inclusive Society. ECIL 2016. Communications in Computer and Information Science, vol 676. Springer, Cham, p. 708-717, 2016.

[ELS 05] ELSTER J., « L'usage stratégique de l'argumentation ». Négociations, n², p. 59-82, 2005.

[ELM 06] ElmBoRg J., « Critical information literacy: Implications for instructional practice », The Journal of Academic Librarianship, Vol. 32, n², p. 192-199, 2006.

[FER 12] FERRAND O., « La révolution médiatique de la condition humaine », Le Débat, Vol. 3, n 170, p.160-174. 2012.

[GAM 89] GAMSON W. A., MODIGLIANI A., « Media discourse and public opinion on nuclear power: A constructionist approach », American journal of sociology, Vol. 95, n¹, p. 1-37, 1989.

[GAU 12] GAUCHAT G., Politicization of science in the public sphere: A study of public trust in the United States, 1974 to 2010. American sociological review, vol. 77, no 2, p. 167-187, 2012.

[GIT 80] GiTLIN T., The whole world is watching. Berkeley, 1980.

[JEH 16] JEHEL S., « Comment s'informent les adolescents des milieux populaires », Diversité, n 185, 2016.

[KOL 11] Koltay T., "The media and the literacies: Media literacy, information literacy, digital literacy », Media, Culture \& Society, vol. 33, n², p. 211-221, 2011.

[LAN 15] LANDRY N., BASQUE J., AGBOBLI C. «Éducation aux médias au Canada: état des savoirs et perspective de recherche en communication », dans A. KIYINDOU (ed.), Éducation aux médias. Nouveaux enjeux, rôles et statuts des acteurs. L'Harmattan, Paris, France, 2015.

[LE 12] LE DEUFF O., «Littératies informationnelles, médiatiques et numériques: de la concurrence à la convergence? », Etudes de communication, $\mathrm{n}^{\circ} 1$, p. 131-147, 2012.

[LE 09] LE DEUFF O., «Penser la conception citoyenne de la culture de l'information », Les Cahiers du numérique, vol. 5, n³, p. 39-49, 2009.

[LEM 07] LEMIEUX C. «À quoi sert l'analyse des controverses? », Mil neuf cent. Revue d'histoire intellectuelle, $n^{\circ} 1$, p.191-212, 2007.

[LOI 09] LOICQ M. «Les enjeux éducatifs de la culture informationnelle », Les cahiers du numérique, vol. 5, n³, p. 7184, 2009.

[MAR 10] MARTY E., Journalismes, discours et publics: une approche comparative de trois types de presse, de la production à la réception de l'information. Thèse de doctorat, Université Toulouse le Mirail - Toulouse II, 2010.

[MER 17] MERZEAU L., « Les fakenews, miroir grossissant de luttes d'influences", InaGlobal, Publié le 19.05.2017, Mis à jour le 31.05.2017, [en ligne], http://www.inaglobal.fr/idees/article/les-fake-news-miroir-grossissant-de-luttes-d-influences-9713

[MON 17] MONOD J. C., « Vérité de fait et opinion politique », Esprit, (Octobre), p. 143-153. 2017.

[OCT 09] OстовRE, S. «Pratiques culturelles chez les jeunes et institutions de transmission: un choc de cultures?», Culture prospective, ${ }^{\circ} 1$, p. 1-8, 2009. 
[OTT 10] OTT B. L., MACK R. L., Critical media studies: An introduction. John Wiley \& Sons, 2010.

Qiu, X., Oliveira, D. F. M., Shirazi, A. S., Flammini, A., \& Menczer, F. (2017). Limited individual attention and online virality of low-quality information. Nature Human Behavior, 1. doi: 10.1038/s41562-017-0132

[QIU 17] QIU X., OLIVEIRA D. F.M., SHIRAZI A. et al. Limited individual attention and online virality of low-quality information. Nature Human Behaviour, vol. 1, no 7, p. 0132, 2017.

[ROO 01a] RoORYCK J. E. C. V. « State of the article: Evidentiality Part I. », GLOT international,vol. 5, n 9, 2001.

[ROO 01b] ROORYCK J. E. C. V. « State of the article: Evidentiality Part II. », GLOT international, vol. 5, n 8, 2001.

[RUB 10] RUBIN V. L., «Epistemic modality: From uncertainty to certainty in the context of information seeking as interactions with texts », Information Processing \& Management, vol. 46, n5, p. 533-540, 2010.

[SAE 15] SAEMmER A., Rhétorique du texte numérique: figures de la lecture, anticipations de pratiques: essai. Presses de l'Enssib, 2015.

[SAE 12] SAEMMER A. «Etude sémio-rhétorique du rôle de l'hypertexte dans le discours journalistique », Médiation et Information, $\mathrm{n}^{\circ} 34,2012$.

[SIM 58] SimOndon G. Du mode d'existence des objets techniques. Eds. Aubier Philosophie (nouvelle édition revue et corrigée, éditée en 2012), (1958).

[SIM 09] SimONNOT B. "Culture informationnelle, culture numérique: au-delà de l'utilitaire», Les Cahiers du numérique, Vol. 5, n³, p. 25-37, 2009.

[SIN 11] SINTOMER Y., Petite histoire de l'expérimentation démocratique: Tirage au sort et politique d'Athènes à nos jours. La Découverte. Paris, 2011.

[SIN 08] SinTOMER Y., « Du savoir d'usage au métier de citoyen? », Raisons politiques, n³, p. 115-133, 2008.

[STI 08] STIEGLER B. Prendre soin: Tome 1, De la jeunesse et des générations, Flammarion, Paris, 2008.

[THE 06] ThEVEnOt L., L’action au pluriel, La Découverte, Paris, 2006.

[TUO 05] TUOMInen K., SAVOlainen R., TAlJa S., « Information Literacy as a Sociotechnical Practice1 ». The Library, vol. $75, \mathrm{n}^{\circ} 3$, p. 329-345, 2005.

[VEN 12] VentURINi T. «Building on faults ». Public Understanding of Science, SAGE Publications, Vol. 21, $\mathrm{n}^{\circ} 7$, p.796-812, 2012.

[VEN 10] Venturini T., « Diving in magma ». Public Understanding of Science, SAGE Publications, Vol. 19, $\mathrm{n}^{\circ} 3$, p.258-273, 2010.

[WIL 96] WILLETT G. « Paradigme, théorie, modèle, schéma : qu’est-ce donc ? », Communication et organisation, $\mathrm{n}^{\circ} 10$, p.1-16, 1996. 